\title{
On the Use of Different Potential Energy Functions in Rare-Gas Cluster Optimization by Genetic Algorithms: Application to Argon Clusters
}

\author{
J. M. C. Marques, ${ }^{*, \dagger}$ F. B. Pereira, ${ }^{\star \S}$ and T. Leitão ${ }^{\S}$ \\ Departamento de Química, Universidade de Coimbra, 3004-535 Coimbra, Portugal, Instituto Superior de \\ Engenharia de Coimbra, Quinta da Nora, 3030-199 Coimbra, Portugal, and Centro de Informática e Sistemas \\ da Universidade de Coimbra (CISUC), 3030-290 Coimbra, Portugal
}

Received: December 19, 2007; Revised Manuscript Received: March 17, 2008

\begin{abstract}
We study the effect of the potential energy function on the global minimum structures of argon clusters arising in the optimization performed by genetic algorithms (GAs). We propose a robust and efficient GA which allows for the calculation of all of the putative global minima of $\operatorname{Ar}_{N}(N=3-78)$ clusters modeled with four different potentials. Both energetic and structural properties of such minima are compared among each other and with those previously obtained for the Lennard-Jones function. In addition, the possibility of obtaining global minima of one potential through local optimization over the corresponding cluster geometry given by other potentials was associated with some structural parameters. The influence of the contribution from the three-body (Axilrod-Teller-Muto) triple-dipole potential (including or not a damping function to describe its correct behavior at smaller interatomic distances) has also been analyzed.
\end{abstract}

\section{Introduction}

A crucial step in the theoretical study of atomic clusters is obtaining the potential energy surface (PES) that establishes the interactions among the atoms. Besides typifying the so-called energetic landscapes, the PES contains all of the relevant information about the system. Basically, the PES may be theoretical or empirically based. In the first case, a set of electronic structure calculations (e.g., ab initio or DFT) covering, at least, the most relevant regions of the coordinate space must be performed, while the second uses information obtained from experiment (e.g., spectroscopic data) in a fitting procedure to a theoretically stated function (which allows for an accurate extrapolation in the configuration space where data is not available). The practical use of ab initio PES implies the interpolation of the calculated points using, for example, a cubic-spline function or fitting them to an adequate function. A common feature of all of these potential functions is their complexity (which increases with dimensionality and accuracy), with many nonlinear terms that become time-consuming during the computational evaluation of the PES. Because of this, the most accurate potentials are avoided in the global minimum search of atomic clusters, and sums of simple pair potentials are usually applied.

In the last years, several theoretical works treating the dimer ${ }^{1-5}$ and trimer ${ }^{6}$ of argon appeared in the literature. In particular, three ${ }^{3-5}$ of those works have calculated the $\mathrm{Ar}_{2}$ potential curve by applying the $\operatorname{CCSD}(\mathrm{T})$ ab initio method with large correlatedconsistent basis sets (which include diffuse orbitals) supplemented by bond functions. In addition, Patkowski et al. ${ }^{3}$ have extrapolated their results to the complete basis set limit to improve the accuracy of the argon-argon potential. It is interesting to notice that the dissociation energy of this new $a b$ initio $\mathrm{Ar}_{2}$ potential is in better agreement with experiment than the benchmark semiempirical potential of Aziz; ${ }^{7}$ this, however,

\footnotetext{
* To whom correspondence should be addressed.E-mail:qtmargue @ ci.uc.pt.

$\dagger$ Departamento de Química, Universidade de Coimbra.

* Instituto Superior de Engenharia de Coimbra, Quinta da Nora.

$\S$ Centro de Informática e Sistemas da Universidade de Coimbra.
}

reproduces better the vibrational quanta. In turn, the availability of very accurate $a b$ initio potentials for the rare gas dimers (e.g., $\mathrm{Ar}_{2}$ ) envisages the possibility of the future calculation of rare gas cluster properties on a "pure" theoretical basis.

The aggregates of argon are usually considered as benchmarks for applying cluster geometry optimization techniques. In this context, Naumkin and Wales ${ }^{8}$ have used the accurate semiempirical $\mathrm{Ar}_{2}$ potential of $\mathrm{Aziz}^{7}$ to calculate the global minima for neutral $\operatorname{Ar}_{N}$ up to $N=55$ and verified that the structures arising are the same as those for the Lennard-Jones (LJ) potential, except at $N=21$ where the stabilities of the two lowest LJ minima are reversed; for $N=21$, the LJ global minimum structure presents $C_{2 v}$ symmetry, while the one obtained from the Aziz potential belongs to the $C_{1}$ point group. In turn, the potential of argon clusters is usually built up as a sum of all of the two-body interaction terms which are modeled by simple, and sometimes inaccurate, functions (e.g., Lennard-Jones); the inclusion in the cluster potential of three-body terms, such as the triple-dipole (Axilrod-Teller-Muto) or even higher-order ones (double-dipole-quadrupole, dipole-quadrupole-quadrupole, and triple-quadrupole), has been done only occasionally. Since the evaluation of accurate two-body potential functions is usually time-demanding, their application to the study of clusters severely restricts the total number of atoms forming the system. Because of this, the simple Rydberg-London $\mathrm{Ar}_{2}$ potential proposed by Cahill and Parsegian ${ }^{9}$ is very appealing for the study of argon clusters. This model function reliably represents the accurate potential of $\mathrm{Aziz}^{7}$ for $\mathrm{Ar}_{2}$, and it also yields accurate second virial coefficients and heats of vaporization. ${ }^{9}$ Indeed, the Rydberg-London (RL) potential for $\mathrm{Ar}_{2}$ is much more accurate than the LJ one while not being much more complicated. Then, one question arises: how different are the global minima of argon clusters modeled by the RL and the LJ potential functions? Moreover, one wonders whether the global minimum structures of the RL model are more reliable than LJ ones to be used as starting points in a local optimization procedure to obtain the corresponding minima of more complex potential energy 
functions (e.g., the ab initio potential of Patkowski et al. ${ }^{3}$ mentioned above or potentials including three-body interactions).

In the present work, we have applied our recently proposed ${ }^{10}$ genetic algorithm (GA) to calculate the global minima of $\mathrm{Ar}_{N}$ $(N=3-78)$ clusters; following the work of Locatelli and Schoen, ${ }^{11,12}$ the GA was also modified to successfully obtain the global minima for some difficult cases in the studied range. The argon clusters have been modeled by four different potentials; two of them include only pair potentials (namely, the RL function ${ }^{9}$ and the ab initio $\mathrm{Ar}_{2}$ potential of Patkowski et al. ${ }^{3}$ ), while the other two include three-body (Axilrod-Teller-Muto) terms in addition to the sum of all RL diatomic interactions. Results obtained by the GA enable us to pursue three major goals: (i) to investigate the energetic and structural differences of global minima of argon clusters modeled by both the RL and $\mathrm{LJ}$ functions and also by the more accurate $\mathrm{ab}$ initio potential of Patkowski et al.; ${ }^{3}$ (ii) to study the effect of the three-body interaction by adding to the sum of the RL two-body potentials all of the long-range triple-dipole (Axilrod-Teller-Muto) interactions, with and without a damping function that accounts for the validity of this term at small interatomic distances; and (iii) to verify if the structures obtained by the GA for clusters modeled with the RL function can be successfully used as starting geometries to obtain the corresponding global minima of more complex functions (such as those involving three-body interactions or the accurate $\mathrm{Ar}_{2}$ potential of Patkowski et al. ${ }^{3}$ ) through local optimization.

The plan of the paper is as follows. In section 3, we outline the computational procedure by presenting the potential energy functions used to model the $\mathrm{Ar}_{N}$ clusters and describing the GA applied in the optimization; in addition, we analyze some difficult cases for the global optimization of argon clusters. A detailed discussion of the results is given in section 3 , while the main conclusions are gathered in section 4 .

\section{Computational Procedure}

2.1. Potential Energy Functions. The potential energy function for the argon cluster may be approximated by

$$
V_{\text {cluster }}=\sum_{i=1}^{N(N-1) / 2} V_{\mathrm{Ar}-\mathrm{Ar}}\left(R_{i}\right)+\sum_{j=1}^{N^{\prime}} V_{\mathrm{Ar}_{3}}\left(\mathbf{R}_{j}\right)
$$

where the first summation includes all two-body terms and the second one refers to the three-body interactions; $\mathbf{R}_{j}$ is the vector of the interatomic distances among the three atoms involved in the $j$ interaction term and $N^{\prime}=\left(\begin{array}{l}N \\ 3\end{array}\right)$. Most of the works on the global optimization of rare gas clusters use the simple LennardJones (LJ) potential to model two-body interactions and do not include three-body contributions. The latter is justified by the fact that three-body terms contribute only with a few percent to the total energy of the cluster. However, such contribution is expected to increase as the number of atoms increases, and hence, for sufficiently large clusters, one expects that it can even change the topology of the global minimum structure. On other side, the use of the LJ function may only be justified by its simplicity and is perhaps the only way, nowadays, to perform calculations for studying extremely large clusters. Nevertheless, it has been shown ${ }^{8}$ that the global minima of argon clusters described by LJ functions may present a different topology from the one obtained with the benchmark $\mathrm{Ar}_{2}$ potential of Aziz, ${ }^{7}$ for example, for $\mathrm{Ar}_{21}$.

In this work, we have applied two types of pair potentials for the global search procedure; one is based on state-of-theart ab initio calculations, while the other is simpler but not so accurate. Thus, the first analytic function is an extension ${ }^{3}$ of the form proposed by Korona et al. ${ }^{13}$

$$
\begin{aligned}
V(R)=\left(A+A^{\prime} R+A^{\prime \prime} / R\right) \exp \left(-\alpha R+\beta R^{2}\right)+ \\
\sum_{n=3}^{8} f_{2 n}(R, b) \frac{C_{2 n}}{R^{2 n}}
\end{aligned}
$$

where $A, A^{\prime}, A^{\prime \prime}, \alpha, \beta$, and $b$ are adjustable parameters, $C_{2 n}$ denotes a dispersion coefficient, and the damping function of Tang and Toennies, ${ }^{14}$ that is, $f_{2 n}(R, b)$, is defined as

$$
f_{2 n}(R, b)=1-\exp (-b R) \sum_{k=0}^{2 n} \frac{(b R)^{k}}{k !}
$$

This potential includes a repulsive exponential term and an attractive truncated damped dispersion component. Note that the additional $A^{\prime}$ and $A^{\prime \prime}$ parameters in eq 2 , not present in the original form of Korona et al., ${ }^{13}$ provide more flexibility in the highly repulsive region of the potential at small internuclear distances. In addition, the introduction of the term containing $A^{\prime}$ is expected to improve the medium-range behavior of the potential. ${ }^{15}$ The values of parameters $A, A^{\prime}, A^{\prime \prime}, \alpha, \beta$, and $b$ have been determined by Patkowski et al. ${ }^{3}$ in a nonlinear leastsquares fit to their $\mathrm{Ar}_{2} \mathrm{CCSD}(\mathrm{T}) / \mathrm{aug}$-cc-pV6Z [supplemented by a $(3 \mathrm{~s} 3 \mathrm{p} 2 \mathrm{~d} 2 \mathrm{f} 1 \mathrm{~g})$ set of midbond functions] ab initio points after adequate ${ }^{16}$ extrapolation to the complete basis set limit (see ref 3 for details). In turn, Patkowski et al. ${ }^{3}$ have used the $\mathrm{ab}$ initio values of Hattig and $\mathrm{Hess}^{17}$ for $C_{6}, C_{8}$, and $C_{10}$ in eq 3 , while the higher-order $\left(C_{12}-C_{16}\right)$ dispersion coefficients have been extrapolated from the previous ones by applying the formula of Thakkar, ${ }^{18}$ all numerical values are given in the original paper. ${ }^{3}$

It has been pointed out by Patkowski et al. ${ }^{3}$ that their $\mathrm{Ar}_{2}$ potential is a refinement of the one of Slavicek et al. ${ }^{4}$ calculated at the same level of theory. Indeed, they have extended the $\operatorname{CCSD}(\mathrm{T})$ calculations to small internuclear distances, besides extrapolating the results to the complete basis set limit (which plays an important role in obtaining an accurate potential). ${ }^{3}$

The other two-body function applied in this work is the simpler Rydberg-London (RL) $\mathrm{Ar}_{2}$ potential that has been proposed by Cahill and Parsegian ${ }^{9}$

$$
V(R)=a \exp (-b R)(1-c R)-\frac{d}{R^{6}+e R^{-6}}
$$

where $a, b, c$, and $e$ are fitting parameters, while $d$ should be fixed at the corresponding London-tail coefficient. Although these parameters can be obtained from ab initio information, Cahill and Parsegian have fitted eq 4 to the Aziz's semiempirical HFDID1 potential for $\mathrm{Ar}_{2}$; the reader is addressed to the paper of Cahill and Parsegian ${ }^{9}$ for the numerical values of the parameters. The use of RL functions to model the two-body interactions in the cluster saves a huge amount of computation time (see below) while expecting to keep a sufficient accuracy of the PES.

Figure 1 shows the Ar-Ar pair-potentials as proposed by Patkowski et al. $^{3}$ (designated as ab initio) and by Cahill and Parsegian 9 (designated as RL); for comparison, the corresponding Lennard-Jones (LJ) curve is also represented. It is apparent from Figure 1 that both ab initio and RL potentials are fairly in agreement, although the former presents a slightly more attractive long-range tail. In contrast, the LJ potential is clearly too attractive for internuclear distances above $\sim 8.5 a_{0}$. As we will see later in this paper, the long-range tail of the LJ potential 


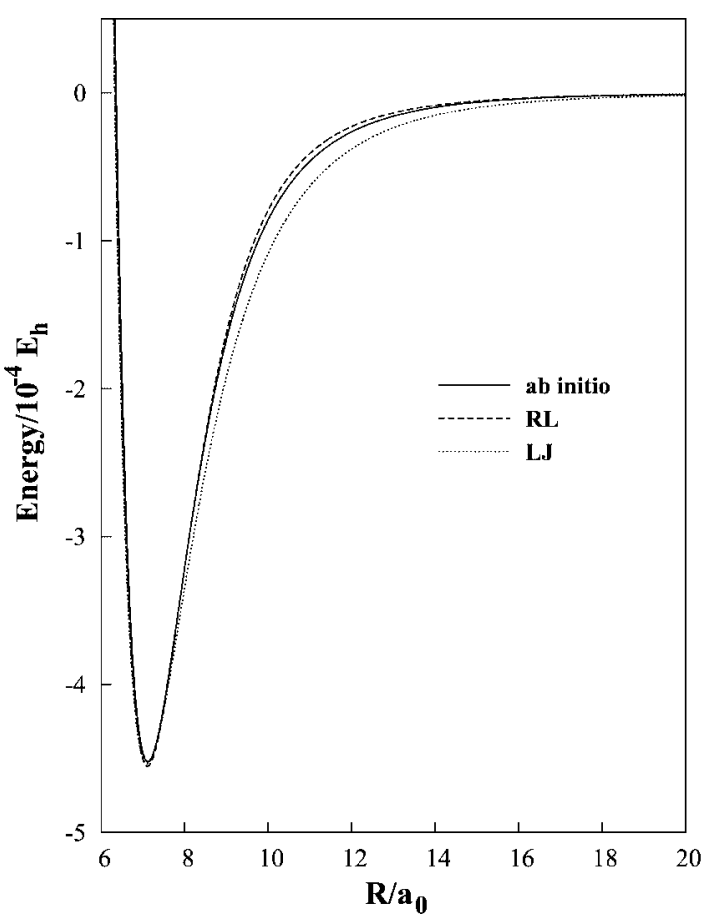

Figure 1. Pair potentials for $\mathrm{Ar}_{2}$ : potential function of eqs 2 and 3, which has been fitted to $a b$ initio data ${ }^{3}$ (solid line), the Rydberg-London function ${ }^{9}$ (dashed line), and the Lennard-Jones potential (dotted line). See the text.

has an influence on the structure and energetics of the global minima of argon clusters.

Concerning the three-body interaction, we employ the wellknown Axilrod-Teller-Muto long-range potential ${ }^{19-21}$

$$
V_{\mathrm{DDD}}\left(R_{1}, R_{2}, R_{3}\right)=\frac{3 C_{\mathrm{DDD}}\left(1+3 \cos \phi_{1} \cos \phi_{2} \cos \phi_{3}\right)}{R_{1}^{3} R_{2}^{3} R_{3}^{3}}
$$

where $R_{1}, R_{2}$, and $R_{3}$ are the internuclear distances defining the triangle whose vertices are atoms 1,2 , and 3 , and $\phi_{i}$ are the corresponding internal angles; the value of $C_{\mathrm{DDD}}$ has been calculated as the average between its upper and lower bounds provided by Standard and Certain, ${ }^{22}$ that is, $C_{\mathrm{DDD}}=176 E_{\mathrm{h}} a_{0}^{9}$. However, eq 5 is valid only in the region where all three interatomic distances are very large. At least two attempts ${ }^{23,24}$ have been made to extend the validity of eq 5 to smaller interatomic distances by the introduction of damping functions. The major differences between the damping function proposed by Varandas $^{23}$ and the one of Sachse et al. ${ }^{24}$ appear only at very small distances that are not relevant for the present study. Then, we have chosen the former to be used in this work. Although higher-order contributions for the three-body interaction should be included, it has been shown that they play a minor role, for example, in the calculated bound states of $\mathrm{Ar}_{3}: 25$ hence, we study in this work only the effect of the Axilrod-Teller-Muto potential (both with and without the damping function proposed in ref 23).

In summary, we have employed in this work four different potential functions to model argon clusters: the sum of pair potentials where the two-body function is the one of Patkowski et al. ${ }^{3}$ (hereafter designated by Pot I) or that proposed by Cahill and Parsegian 9 (Pot II); in the other two functions, the twobody interactions are also described by eq 4 , while the threebody contribution is assumed to follow eq 5 only (Pot III) or
TABLE 1: Average Computation Time Spent in the Evaluation of the Potential and the Derivatives for the LJ and the Four Functions Used in This Work ${ }^{a}$

\begin{tabular}{lcc}
\hline & \multicolumn{2}{c}{ average computation time $^{b}$} \\
\cline { 2 - 3 } function & potential & derivatives \\
\hline LJ $^{c}$ & 100 & 12960 \\
Pot I & 4060 & 21070 \\
Pot II & 330 & 13450 \\
Pot III & 3830 & 242050 \\
Pot IV & 43430 & 312520
\end{tabular}

${ }^{a}$ In all cases, the average time corresponds to a calculation on a ATHLON 64 X2 $3800+2.0 \mathrm{GHz}$ by performing 1000 evaluations of the potential function or derivatives of $\mathrm{Ar}_{78}$, whose coordinates have been generated randomly. ${ }^{b}$ The average computation times are given in internal CPU units. ${ }^{c} \mathrm{LJ}$ stems for the summation of all Lennard-Jones pair potentials of the $\mathrm{Ar}_{78}$ cluster.

eq 5 damped for small internuclear distances as described above (Pot IV). Table 1 presents the average computational times required to calculate all of these potentials and their derivatives for $\mathrm{Ar}_{78}$. In terms of the CPU time consumed for the calculation of the potential, the following order is presented: Pot II $<$ Pot III $<$ Pot I $<$ Pot IV. In contrast, the order for the computation

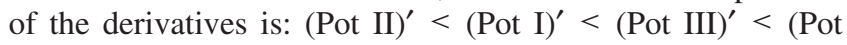
IV $)^{\prime}$. It is worth noting that the computation of Pot I is slightly more expensive than that of Pot III, but the average CPU time for the calculation of the corresponding derivatives follows the reverse order. This may be attributed to the fact that Pot III uses simple RL pair potentials (note the extremely short CPU time of Pot II that uses only RL functions), while Pot I is a sum of the more complicated functions given by eqs 2 and 3; however, the two-body contribution for the CPU time is overwhelmed by the calculation of three-body derivatives. Finally, since the optimization procedure uses both the potential and derivatives (see section 2.2), one expects the average CPU time needed for the global minimum search to follow the aforementioned order for the computation of derivatives.

2.2. Genetic Algorithm for Global Optimization. The genetic algorithm (GA) used in the present work has been recently proposed by us ${ }^{10}$ and has shown to perform well for Morse clusters. The main steps carried out by the GA are represented in the flowchart of Figure 2. Although it may be seen as analogous to other GAs already published in the literature (ref 26 and references therein), our search procedure presents some subtle differences. Basically, it is a generational hybrid-type algorithm which we describe in the following.

The search procedure begins with the generation of 100 individuals (cluster structures) by randomly choosing the corresponding $3 \mathrm{~N}$ Cartesian coordinates of the $N$ atoms forming the cluster; the coordinates are allowed to have values between 0 and $8.5 N^{1 / 3},{ }^{27}$ but only internuclear distances larger than $5 a_{0}$ are accepted because the potential becomes too repulsive when two atoms approach close to each other. Then, as represented in Figure 2, all of the 100 individuals of the population are subjected to local optimization by applying the BroydenFletcher-Goldfarb-Shanno-limited memory quasi-Newton (LBFGS) method ${ }^{28,29}$ (a maximum of 500 iterations are allowed for each local optimization). After local optimization, the fitness of each individual is evaluated using the appropriate potential function. The GA adopts a fully generational approach, that is, in each generation, the whole population is replaced by its offspring. The new set of solutions is obtained after a sequence of steps is performed. First, tournament selection (with a tourney size of 5) chooses 100 parents, and afterward, the standard 


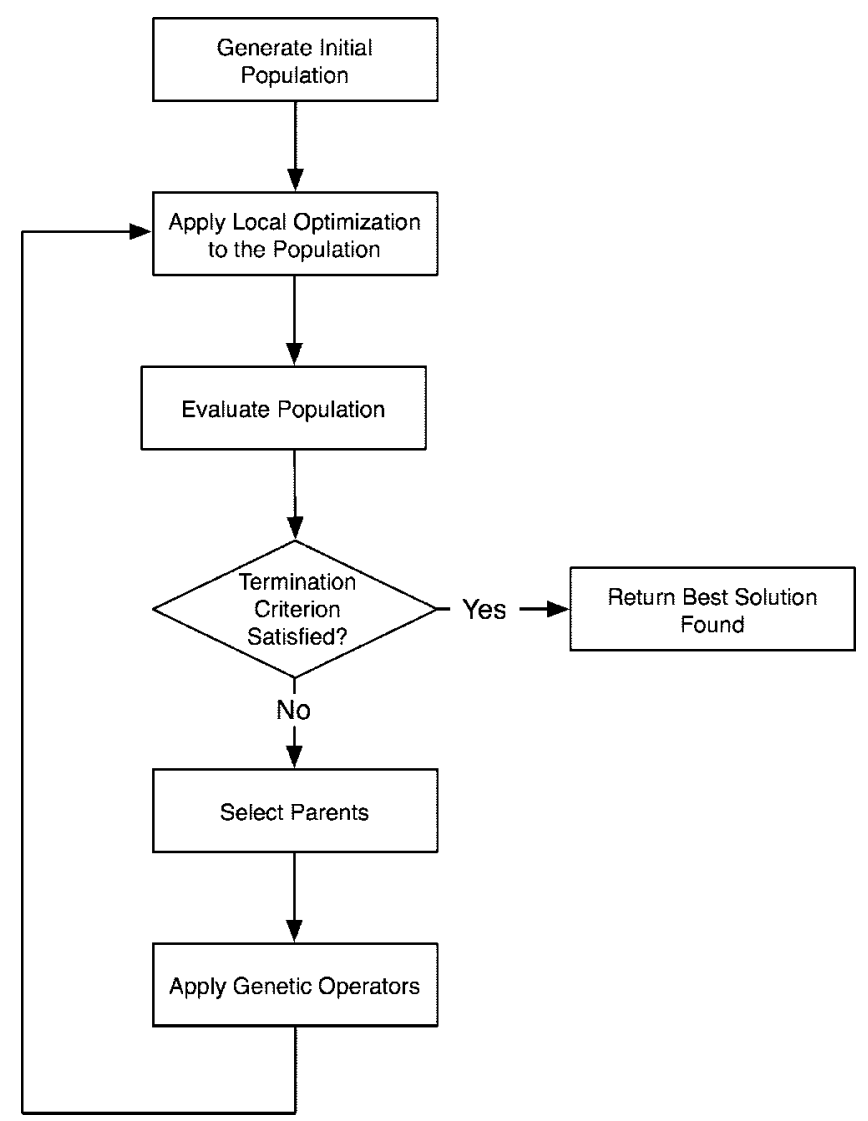

Figure 2. Flowchart representing the main steps of the GA applied in the present work. See the text.

genetic operators are applied to the selected individuals. Cut and splice crossover, proposed by Deaven and Ho, ${ }^{30}$ is applied with a probability of 0.7 to the 50 pairs of selected structures. Then, a mutation operator is used to guarantee a certain degree of diversity in the population. In this study, we have applied Sigma mutation with a probability of 0.1 to each one of the atoms that compose the individuals resulting from crossover. We further note that the application of this operator to an atom consists in its displacement along the three Cartesian axes by a random amount (whose values are sampled from a standard normal distribution) proportional to $\sigma$; in this study, we have selected $\sigma=0.1 \times\left(8.5 N^{1 / 3}\right)$. The descendants that result from the application of the genetic operators are locally optimized using the aforementioned procedure and, then, evaluated. We emphasize at this stage that the hybrid GA was tailored to maximize performance, which is achieved by ensuring high locality features in the algorithm. We have shown that high locality may be achieved through the application of a moderate disruptive mutation operator followed by local optimization. ${ }^{10,31}$ This GA implements an improved elitist strategy. During the optimization, it classifies individuals into four different classes according to their momenta of inertia: \{oblate, spherical, prolate, asymmetric $\}$. Then, whenever the process of creating a new population is complete, the elite operator is applied to members of each class, ensuring that the quality of the best individual with a given rotational symmetry does not decrease along the generations. This approach guarantees the preservation of the best solutions found so far and implements a basic mechanism for the preservation of diversity. The new population of 100 individuals substitutes the old one, and all of the process is repeated up to the generation where the number of calls to the evaluation function reaches 500000 . The best individual obtained within 30 runs of the described GA is assigned as the global minimum structure of a given $N$-atom cluster.

2.3. Difficult Cases for Global Optimization. We address here the problems one met in the global search of argon clusters for some sizes (i.e., values of $N$ ) by applying the simple GA described in the previous subsection. It is well-known from the literature (ref 32 and references therein) that the global optimization of atomic clusters (with Lennard-Jones or, especially, Morse potentials) becomes particularly hard for some nuclearities; additional difficulties for global optimization arise due to the general increase in the number of local minima with $N .^{33-35}$ The size-specific difficulties are essentially related to the particular topography features of the potential energy surface (PES). Whereas for most of the Lennard-Jones clusters the topography of the PES is characterized by a single funnel coming from the high-energy regime to the low-energy icosahedral-based minima ${ }^{36}$ (which contains the global minimum), the $N=38$ and 75-77 clusters (just to mention those in the range studied here) present, as well, a much narrower funnel leading to the global minimum. ${ }^{37,38}$ Thus, the probability of reaching the basin of attraction of the global minimum is extremely low in such cases, while the large energy barriers between the two funnels prevent traditional searching methods (e.g., simulated annealing) from being successful. Indeed, even more powerful approaches like the "basin-hopping" 39 or GAs 30,40 have difficulties to find minima for those double-funnel clusters. ${ }^{41}$

Since there is no table of putative global minima for the potential functions applied in this work, we have used for comparison those obtained by local minimization of the corresponding LJ clusters. The putative global minimum for a given argon cluster modeled by each potential function is not reached in the GA optimization whenever the energy of the optimum so obtained is higher than that arising from the local minimization of the corresponding LJ structure. Specifically, besides failing to find out the global minima of argon clusters in the range of $N=75-77$ for all of the potentials (Pot I-Pot IV), the GA described in section 2.2 was not successful for $N=65$ (with Pot I) and 78 (with Pot I, Pot III, and Pot IV). In contrast, our GA was quite efficient in discovering the global minima of all of the other clusters, including the $N=38$ one (although in this case, the rate of success was only $\sim 10 \%$ ).

In the past few years, a couple of approaches ${ }^{11,12,42}$ were developed to deal with this double-funnel problem in a more efficient way. The technique introduced by Hartke ${ }^{42}$ uses a parameter which controls the diversity of structural types in the population of the GA, preventing it from generating only icosahedral structures. In turn, Locatelli and Schoen ${ }^{11,12}$ have proposed a transformation in the potential energy surface, so that the nonicosahedral global minima are much more likely to be found. It consists of applying a two-phase local optimization, comprising a minimization of the modified function followed by the search of the corresponding minimum in the "true" PES. The modified PES includes two penalty terms and can be represented as follows

$$
V_{\text {cluster }}^{\text {mod }}=V_{\text {cluster }}+\sum_{k}\left\{\mu R_{k}+\beta\left[\max \left(0, R_{k}^{\prime 2}-D^{2}\right)\right]^{2}\right\}
$$

where the first term is the "true" PES and the penalty parameters $\mu, \beta$, and $D$ have to take non-negative values; $D$ is an underestimate of the diameter of the cluster and $R_{k}{ }^{\prime}$ is a modification of the internuclear distance $R_{k}$ (between atoms $i$ and $j$ ) 


$$
R_{k}{ }^{\prime}=\left[w_{1}\left(x_{i}-x_{j}\right)^{2}+w_{2}\left(y_{i}-y_{j}\right)^{2}+w_{3}\left(z_{i}-z_{j}\right)^{2}\right]^{1 / 2}
$$

where $(x, y, z)$ are Cartesian coordinates and the weights $w_{1}$, $w_{2}$, and $w_{3}$ may have different values between 0 and 1 , so that one shape may be favored in relation to another; for example, by choosing different weights for the three Cartesian coordinates, ellipsoidal shapes may be favored in relation to spherical ones. ${ }^{12}$ It is important to note in eq 6 that the second term (first in the summation) should give stronger penalty to internuclear distances greater than $R_{\mathrm{e}}$ (the equilibrium distance). This term has a spherical compression effect on the cluster since it acts on all pairs of atoms ${ }^{12}$ and contributes to reduce the number of minima and transition states on the PES. ${ }^{43}$ In contrast, the last term in eq 6 strongly penalizes internuclear distances greater than the estimated diameter of the cluster $(D)$ but has no influence on pairs of atoms close to each other. ${ }^{11}$ This shape-type penalty term is crucial for discovering the most difficult Lennard-Jones clusters. ${ }^{11,12}$

On the basis of its success for LJ and Morse clusters, ${ }^{11,12,44,45}$ we expect that the two-phase optimization method ${ }^{11,12}$ described above is able to increase the performance of our GA in discovering the global minima of the "difficult" $\mathrm{Ar}_{N}$ clusters. We note that the implementation of the two-phase optimization procedure in our GA is straightforward (only the local optimization step of the flowchart in Figure 2 needs to be modified), being that the transformation of the PES (eqs 6 and 7) is applied only to the two-body potentials; even in the cases of Pot III and Pot IV, we expect this transformation to be efficient since the magnitude of the three-body terms is very small in comparison with that of two-body ones. As a testing ground, we have applied this new GA to search the global minimum for $\mathrm{Ar}_{38}$. The rate of success increases from $\sim 10 \%$ (as mentioned above) to $100 \%$ for all of the potentials. Moreover, the improved version of the GA allows for discovering of the putative global minima of all argon clusters that could not be reached by the simple algorithm described in the previous subsection. For completeness, we give in Table 2 the values of parameters of eqs 6 and 7 used for the global optimization of each "difficult" argon cluster. It should be noted that the parameters given in Table 2 allowed us to find out the global minimum at least once in 30 runs of the GA, but they have not been tuned to maximize the success rate; this point may be addressed in a future work.

\section{Results and Discussion}

Both the evolutionary algorithm described in section 2.2 and its improved version (section 2.3) have been applied to obtain the putative global minima of $\operatorname{Ar}_{N}(N=3-78)$ clusters as modeled by Pot I, Pot II, Pot III, and Pot IV presented in section 2.1. The energies of the global minima obtained for the four potential functions are given in Table 3. Note that, for each $N$, the largest difference in the energy of the global minima among the four potential functions never exceeds $5 m E_{\mathrm{h}}(\sim 2.7 \%$ of the total energy). In general, this difference tends to increase with $N$, and it is largest between Pot I and Pot III. Although not shown in Table 3, the corresponding difference between the global minima of Pot II and those obtained ${ }^{8}$ by using the Aziz potential $^{7}$ varies from $\sim-0.5 \%$ for $N=3$ up to $\sim 0.9 \%$ for $N$ $=55$; for $N \geq 10$, the global minima from the Aziz potential become deeper than those of Pot II. These small differences are not surprising if one has in mind that the RL potential was fitted to the Aziz potential (see section 2.1). Also given in Table 3 is the symmetry point group of each minimum. Most of the argon clusters in the range of $3 \leq N \leq 78$ have global minima structures belonging to the same symmetry point group, which
TABLE 2: Parameters of Equations 6 and 7 Used in the Global Minimum Search of Argon Clusters That Are Hard to Optimize; See the Text

\begin{tabular}{lllllll}
\hline cluster & $\mu / E_{\mathrm{h}} a_{0}^{-1}$ & $\beta / E_{\mathrm{h}} a_{0}^{-4}$ & $D / a_{0}$ & $\omega_{1}$ & $\omega_{2}$ & $\omega_{3}$ \\
\hline $\mathrm{Ar}_{38}$ & $6.4 \times 10^{-6}$ & $1.78 \times 10^{-7}$ & 20 & 1 & 1 & 1 \\
$\mathrm{Ar}_{65}$ & 0 & $4.52 \times 10^{-4}$ & 12 & 1 & 0.85 & 0.65 \\
$\mathrm{Ar}_{75}$ & $3.2 \times 10^{-5}$ & $4.52 \times 10^{-4}$ & 20 & 1 & 0.7 & 0.7 \\
$\mathrm{Ar}_{76}$ & $3.2 \times 10^{-5}$ & $4.52 \times 10^{-4}$ & 20 & 1 & 0.7 & 0.4 \\
$\mathrm{Ar}_{77}$ & $3.2 \times 10^{-5}$ & $4.52 \times 10^{-4}$ & 12 & 1 & 0.75 & 0.5 \\
$\mathrm{Ar}_{78}$ & $3.2 \times 10^{-5}$ & $4.52 \times 10^{-4}$ & 12 & 1 & 0.75 & 0.5
\end{tabular}

is independent of the potential used; one exception arises at $N$ $=78$, for which Pot I, Pot III, and Pot IV lead to $C_{1}$ structures while Pot II presents a global minimum with $C_{s}$ symmetry. Thus, the specific features of these potentials appear to have little influence on the topology of argon clusters up to $\mathrm{Ar}_{78}$. The symmetry point groups are in agreement with those obtained by Naumkin and Wales ${ }^{8}$ for argon clusters up to $\mathrm{Ar}_{55}$, which have been modeled by the benchmark potential of Aziz. ${ }^{7}$ Excluding $\operatorname{Ar}_{21}\left(C_{1}\right), \operatorname{Ar}_{67}\left(C_{2}\right), \operatorname{Ar}_{69}\left(C_{s}\right)$, and $\operatorname{Ar}_{78}\left(C_{1}\right.$ in case of Pot I, Pot III, and Pot IV), the agreement is also apparent between the present structures and those obtained with the simple Lennard-Jones potential; ${ }^{46}$ the corresponding symmetry point groups for LJ clusters are $C_{2 v}, C_{s}, C_{5 v}$, and $C_{s}$, respectively.

3.1. Energetic and Structural Trends of Pair Potentials. The average binding energy can be calculated from Table 3 through the expression ${ }^{47}$

$$
\left\langle E_{b}\right\rangle=-V_{\text {cluster }}(N) / N
$$

where $V_{\text {cluster }}(N)$ is the global minimum energy of the cluster with $N$ atoms. Another important quantity is the sublimation energy, that is, the energy required to remove an atom from the cluster

$$
\Delta E_{\mathrm{sub}}=E(N)-E(N-1)
$$

where $E(N)=-V_{\text {cluster }}(N)$ and similarly for $E(N-1)$. Although both $\left\langle E_{b}\right\rangle$ and $\Delta E_{\text {sub }}$ may give important information about the energetics of the clusters, the relative stability is better taken into account by calculating the second difference in energy, that is

$$
\Delta_{2} E=2 E(N)-E(N-1)-E(N+1)
$$

It is this quantity that is generally compared with experimental mass spectral intensities.

We represent in Figure 3 the average binding energy [panel (a)], the sublimation energy [panel (b)], and the second energy difference [panel (c)] as a function of the size of the cluster. We include in Figure 3 the results calculated with Pot I and Pot II of the present work as well as those obtained with the LJ clusters. ${ }^{46}$ As a general trend for all three potentials, one observes that the average binding energy [panel (a)] increases rapidly with the number of atoms in the cluster for $N \leq 13$; then, as $N$ increases, the slope of the curve tends to decrease. Particularly interesting in this figure is the apparent coincidence between the curves for Pot I and Pot II, while the average binding energy from the Lennard-Jones potential diverges as $N$ increases. This may be related to the fact that the $\mathrm{LJ}$ potential is more long-range than the $\mathrm{ab}_{\text {initio }}{ }^{3}$ and the Rydberg-London ${ }^{9}$ ones (see Figure 1). We further note the appearance of local regions of enhanced stability (small concavities) all over of the curves and, in particular, for $N=13$ and 55 (in this case, the curve corresponds to a maximum), which are known to be stable icosahedral structures. This is especially obvious from the peaks 
TABLE 3: Energies (in $m E_{\mathrm{h}}$ ) and Symmetries of the Global Minima of $\operatorname{Ar}_{N}(3 \leq N \leq 78)$ Obtained with the Four Potentials Investigated in This Work

\begin{tabular}{|c|c|c|c|c|c|}
\hline$N$ & symmetry & Pot I & Pot II & Pot III & Pot IV \\
\hline 3 & $D_{3 h}$ & -1.35691 & -1.36631 & -1.36111 & -1.36167 \\
\hline & $T_{d}$ & -2.71382 & -2.73263 & -2.71184 & -2.71410 \\
\hline & $D_{3 h}$ & -4.10349 & -4.12767 & -4.09030 & -4.09417 \\
\hline & $O_{h}$ & -5.67757 & -5.69830 & -5.63884 & -5.64390 \\
\hline & $D_{5 h}$ & -7.39167 & -7.42172 & -7.33724 & -7.34528 \\
\hline 8 & $C_{s}$ & -8.84616 & -8.87323 & -8.77065 & -8.78014 \\
\hline & $C_{2 v}$ & -10.73713 & -10.76437 & -10.63165 & -10.64369 \\
\hline 10 & $C_{3 v}$ & -12.62101 & -12.64608 & -12.48225 & -12.49684 \\
\hline 11 & $C_{2 v}$ & -14.50991 & -14.53074 & -14.33495 & -14.35213 \\
\hline 12 & $C_{5 v}$ & -16.76436 & -16.78449 & -16.54247 & -16.56349 \\
\hline 13 & $I_{h}$ & -19.54973 & -19.57116 & -19.26499 & -19.29127 \\
\hline 14 & $C_{3 v}$ & -21.06413 & -21.07550 & -20.75087 & -20.77831 \\
\hline 15 & $C_{2 v}$ & -23.00568 & -23.00995 & -22.65482 & -22.68446 \\
\hline 16 & $C_{s}$ & -24.94037 & -24.93549 & -24.54913 & -24.58091 \\
\hline 17 & $C_{2}$ & -26.87660 & -26.86217 & -26.44494 & -26.47877 \\
\hline 18 & $C_{5 v}$ & -29.09673 & -29.07731 & -28.61207 & -28.64970 \\
\hline 19 & $D_{5 h}$ & -31.76234 & -31.75067 & -31.22284 & -31.26573 \\
\hline 20 & $C_{2 v}$ & -33.67800 & -33.65236 & -33.09359 & -33.13822 \\
\hline 21 & $C_{1}$ & -35.58915 & -35.55302 & -34.96343 & -35.01015 \\
\hline 22 & $C_{s}$ & -37.76036 & -37.71458 & -37.07943 & -37.12935 \\
\hline 23 & $D_{3 h}$ & -40.34019 & -40.30133 & -39.60407 & -39.65881 \\
\hline 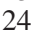 & $C_{s}$ & -42.23723 & -42.18230 & -41.45441 & -41.51085 \\
\hline & $C_{s}$ & -44.33858 & -44.27194 & -43.50112 & -43.56047 \\
\hline 26 & $T_{d}$ & -46.84264 & -46.78212 & -45.94903 & -46.01295 \\
\hline 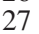 & $C_{2 v}$ & -48.82729 & -48.75820 & -47.89565 & -47.96172 \\
\hline 28 & $C_{s}$ & -50.86546 & -50.77644 & -49.87321 & -49.94158 \\
\hline 29 & $D_{3 h}$ & -53.24880 & -53.16414 & -52.19961 & -52.27217 \\
\hline 30 & $C_{2 v}$ & -55.29208 & -55.19645 & -54.20110 & -54.27600 \\
\hline & $C_{s}$ & -57.62955 & -57.3 & -56.38502 & -56.45350 \\
\hline 32 & $C_{2 v}$ & -60.20651 & -59.91201 & -58.89066 & -58.96263 \\
\hline 33 & $C_{s}$ & -62.39393 & -62.07399 & -61.01328 & -61.08725 \\
\hline 34 & $C_{2 v}$ & -64.57976 & -64.23434 & -63.13442 & -63.21040 \\
\hline 35 & $C_{1}$ & -67.03933 & -66.68158 & -65.53637 & -65.61588 \\
\hline 36 & $C_{s}$ & -69.61840 & -69.24240 & -68.04338 & -68.12638 \\
\hline 37 & $C_{1}$ & -71.80522 & -71.40368 & -70.16554 & -70.25053 \\
\hline 38 & $O_{h}$ & -74.64036 & -74.14214 & -72.87512 & -72.95546 \\
\hline 30 & $C_{5 v}$ & -77.34581 & -76.90678 & -75.55246 & -75.64533 \\
\hline 40 & $C_{s}$ & -79.53493 & -79.07027 & -77.67692 & -77.77175 \\
\hline 41 & $C_{s}$ & -81.74517 & -81.25195 & -79.81821 & -79.91505 \\
\hline & $C_{s}$ & -84.20850 & -83.70233 & -82.22277 & -82.32308 \\
\hline 43 & $C_{s}$ & -86.78679 & -86.26216 & -84.72831 & -84.83215 \\
\hline 4 & $C_{1}$ & -89.00626 & -88.45184 & -86.87715 & -86.98297 \\
\hline 45 & $C_{1}$ & -91.56036 & -90.98415 & -89.35680 & -89.46565 \\
\hline 46 & $C_{2 v}$ & -94.53076 & -93.93973 & -92.24851 & -92.36218 \\
\hline 47 & $C_{1}$ & -96.75126 & -96.13017 & -94.39813 & -94.51378 \\
\hline 48 & $C_{s}$ & -99.32511 & -98.6 & -96.89172 & -97.01039 \\
\hline 49 & $C_{3 v}$ & -102.29061 & -101.62953 & -99.77864 & -99.90215 \\
\hline 0 & $C_{s}$ & -104.54794 & -103.85245 & -101.95835 & -102.08386 \\
\hline 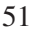 & $C_{2 v}$ & $-10^{7}$ & 52088 & 56189 & -104.68988 \\
\hline 52 & $C_{3 v}$ & -110.24273 & -109.48949 & -107.46464 & -107.59743 \\
\hline 53 & $C_{2 v}$ & -113.22743 & -112.45637 & -110.36561 & -110.50320 \\
\hline 54 & $C_{5 v}$ & -116 & -11 & -11 & -11 \\
\hline 55 & $I_{h}$ & -119.22265 & -118.41203 & -116.18919 & -116.33615 \\
\hline 56 & $C_{3 v}$ & 1955 & 17804 & -117.92503 & -118.07344 \\
\hline 57 & $C_{s}$ & -123 & -12 & -119.88858 & -12 \\
\hline 58 & $C_{3 v}$ & -125.60273 & -124.72684 & -122.39976 & -122.55282 \\
\hline & $C_{2 v}$ & -127.81305 & -12 & -12 & 68788 \\
\hline 60 & $C_{s}$ & -130.41620 & -12 & -12 & -12 \\
\hline 61 & $C_{2 v}$ & -133.01619 & -132.06543 & -129.59413 & -129.75569 \\
\hline & $C_{s}$ & 20277 & 21853 & 69988 & -131.86410 \\
\hline & $C_{1}$ & -137.80002 & 9403 & 2641 & -134.39352 \\
\hline 64 & $C_{s}$ & -140.39436 & -139.36662 & -136.74998 & -136.91999 \\
\hline & $C_{2}$ & -142.58329 & 216 & 5844 & 3107 \\
\hline & $C_{1}$ & -14 & -14 & -1 & -14 \\
\hline 67 & $C_{2}$ & -147.77031 & -146.66582 & -143.90431 & -144.08270 \\
\hline & $C_{1}$ & 28603 & 810 & 3323 & -146.51750 \\
\hline & $C_{s}$ & 3695 & & 0145 & -149.08870 \\
\hline 10 & $C_{5 v}$ & -155.91957 & -154.72271 & -151.77176 & -151.96167 \\
\hline & $C_{5 v}$ & -158.62526 & -157.42369 & -154.40571 & -154.60058 \\
\hline & $C_{s}$ & 83553 & -159.60751 & -156.55098 & -156.74768 \\
\hline 13 & $C_{s}$ & -163.36224 & -162.10086 & -158.98772 & -159.18781 \\
\hline & $C_{s}$ & -165.94604 & -164.66304 & 50118 & -161.70410 \\
\hline & $D_{5 l}$ & 79947 & -167.30390 & -164.19232 & -164.38079 \\
\hline & $C_{s}$ & -171.03976 & -169.51807 & -166.36214 & -166.55331 \\
\hline & & -173.67393 & -172.12727 & -168.92252 & -169.11653 \\
\hline & & & 39790 & -1 & -171.28 \\
\hline
\end{tabular}

${ }^{a} C_{1}$ is for Pot I, Pot III, and Pot IV, while the minimum for Pot II presents $C_{s}$ symmetry.
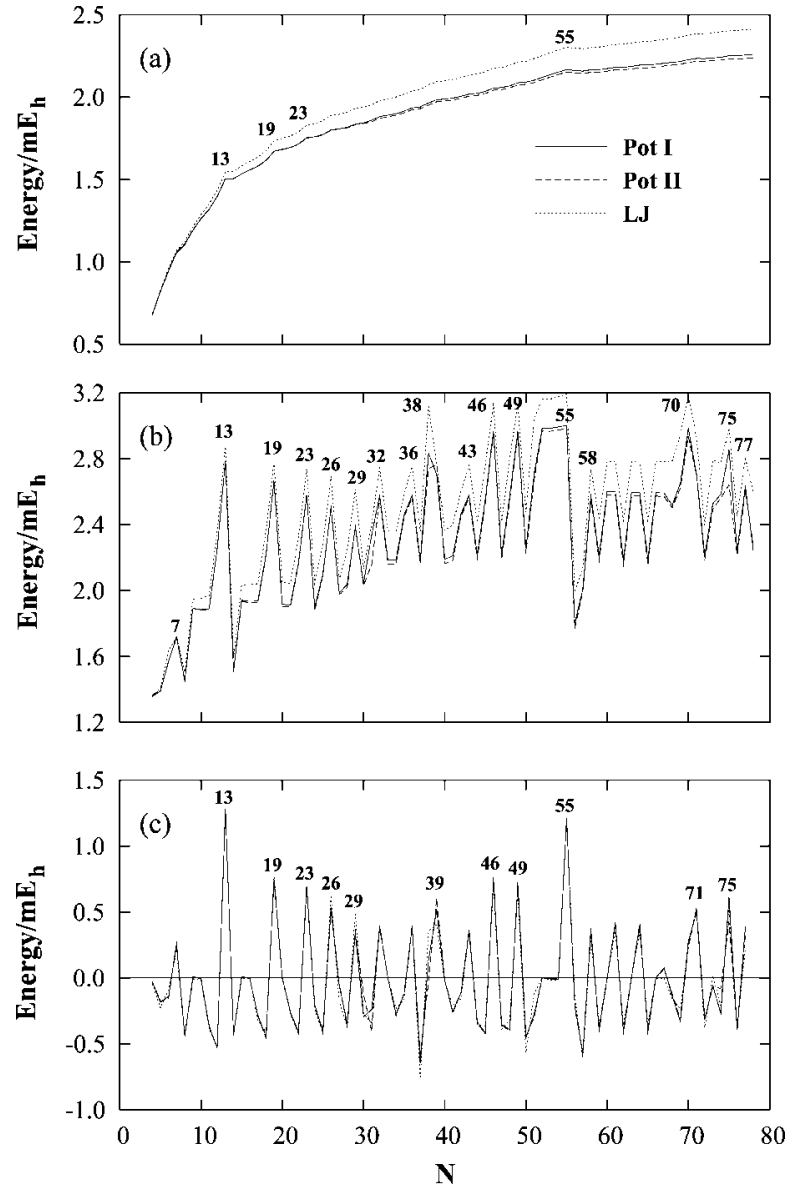

Figure 3. Average binding energy (a), sublimation energy (b), and second energy difference (c) as a function of the size of the cluster. The lines represent the results for Pot I (solid), Pot II (dashed), and Lennard-Jones (dotted). The most relevant line features are pointed out by the corresponding numbers of $N$ in panels (a) and (b). In turn, the numbers in panel (c) mark the peaks associated to clusters with a minimum coordination number of 6 . See the text.

(maxima) shown in panels (b) and (c), which tend to be associated with structures with a high relative stability.

It is clear from Figure $3 b$ that the sublimation energies calculated from Pot I and Pot II are essentially the same for all clusters considered in this work. Conversely, the sublimation energies obtained with the Lennard-Jones potential tend to overestimate those of Pot I and Pot II, especially as $N$ increases; in any case, all potentials anticipate the same peaks. We note, among others, the high values of the sublimation energy for the $N=13$ and 55 icosahedral structures, as well as the $N=$ 38 octahedral cluster. This pattern may be associated with the high stability of the icosahedral structures, and in fact, one obtains for these clusters similar peaks in the curves of the second energy difference shown in Figure 3c, while the maximum in the sublimation energy for $\mathrm{Ar}_{38}$ must be due to the relative high instability of $\mathrm{Ar}_{37}$; indeed, $\mathrm{Ar}_{39}$ is relatively more stable than $\mathrm{Ar}_{38}$, as can be seen from Figure $3 \mathrm{c}$. In addition, it is especially interesting to observe in Figure $3 \mathrm{~b}$ that the nearicosahedral $\mathrm{Ar}_{52}, \mathrm{Ar}_{53}$, and $\mathrm{Ar}_{54}$ structures present sublimation energies which are only slightly below the value for $\mathrm{Ar}_{55}$.

Figure $3 \mathrm{c}$ shows for the second energy difference a set of maxima corresponding to structures that present a relative stability with respect to their immediate neighbors (the so-called "magic numbers"). In this set, we can find a great variety of cluster structures, the highest peaks being those for $\mathrm{Ar}_{13}$ and $\operatorname{Ar}_{55}$ (Mackay icosahedra). Also, important peaks appear for $\operatorname{Ar}_{19}$ 
(two fused centered icosahedra), $\mathrm{Ar}_{23}$ (triple icosahedra), $\mathrm{Ar}_{26}$ (quadruple icosahedra), $\mathrm{Ar}_{29}, \mathrm{Ar}_{39}, \mathrm{Ar}_{46}, \mathrm{Ar}_{49}, \mathrm{Ar}_{71}$, and $\mathrm{Ar}_{75}$ (Marks decahedra). Note that these clusters are relatively stable because they have the surface atoms highly coordinated (in all of these cases, the minimum coordination number is 6). Moreover, other minor peaks are visible in Figure $3 \mathrm{c}$ for intermediate nuclearities whose structures present a minimum coordination number smaller than 6 . Excluding the very small peak at $N=67$ that is not present in the case of $\mathrm{LJ}$, the other ones are fairly predicted by Pot I and Pot II.

A better way to understand the relation between the form of the pair potential and the global minimum energy of the cluster may be achieved by partitioning it into three components ${ }^{48}$

$$
V_{\text {cluster }}=E_{\text {nn }}+E_{\text {strain }}+E_{\mathrm{n}-\mathrm{nn}}
$$

where $E_{\mathrm{nn}}$ is the energy obtained by assuming that all distances between nearest-neighbor atoms are equal to the equilibrium distance $\left(R_{\mathrm{e}}\right)$ of the corresponding diatomic potential, while $E_{\text {strain }}$ is the component accounting for the bonding distances being strained in relation to $R_{\mathrm{e}} ; E_{\mathrm{n}-\mathrm{nn}}$ is the energetic contribution of all non-nearest-neighbor interactions (i.e., associated with large distances) between the atoms of the cluster. Though the separation in three different components may be helpful for the present analysis, one should have in mind that those terms in eq 11 are not completely independent for obtaining the global minimum structure as discussed below. We represent in Figure 4 these three components of the energy for Pot I, Pot II, and LJ. It is clear from this figure that the energy due to the nearest-neighbor contacts $\left(E_{\mathrm{nn}}\right)$ is the most important component among those in eq 11. It is worth noting from Figure 4a that the $E_{\mathrm{nn}}$ curves for the three potentials are nearly coincident, which is an indication of both similar well depths and essentially the same number of nearest-neighbor distances for each $N$. In contrast, the differences among the potentials are well patented in the curves of the strain energy [panel (b)] and $E_{\mathrm{n}-\mathrm{nn}}$ [panel (c)]. In both cases, all of the curves present a similar behavior with $N$, but the differences among the potentials studied increase with the number of atoms in the cluster and are larger between LJ and the other two functions than between Pot I and Pot II. Moreover, LJ clusters tend to be less (more) strained than those modeled with Pot I and Pot II for $N \leq 30(N>30)$, which may be a consequence of the longer range of the LJ pair potential. This factor acts in two opposite ways: on one side, $E_{\text {strain }}$ decreases with the increasing range of the potential for a given geometry ${ }^{49}$ because the curvature of the potential is less narrow; on the other side, the contribution from $E_{\mathrm{n}-\mathrm{nn}}$ becomes more important as $N$ increases (especially for LJ clusters, as shown in Figure 4c), which leads to more-strained structures (i.e., $E_{\text {strain }}$ increases essentially due to compression). The balance of these two factors occurs for $N$ around 30 and explains the behavior displayed in Figure 4b.

It is apparent from Figure $4 \mathrm{a}$ that the values of $E_{\mathrm{nn}}$ tend to decrease (i.e., to be more negative) with increasing $N$ since the number of nearest-neighbor distances is expected to rise with the nuclearity of the cluster. Exceptions to this behavior occur for $N=30-31$ and 74-75, which is due to a decrease in the number of nearest-neighbor distances for $\mathrm{Ar}_{31}$ and $\mathrm{Ar}_{75}$ in relation to $\mathrm{Ar}_{30}$ and $\mathrm{Ar}_{74}$, respectively. It is interesting to observe in Figure $4 \mathrm{~b}$ the abrupt fall off of the strain energy for those nuclearities, which is a likely factor to determine the aforementioned decrease in the number of nearest-neighbor distances. Indeed, the strain energy increases essentially up to $N=30$ so that the clusters may accomplish the highest
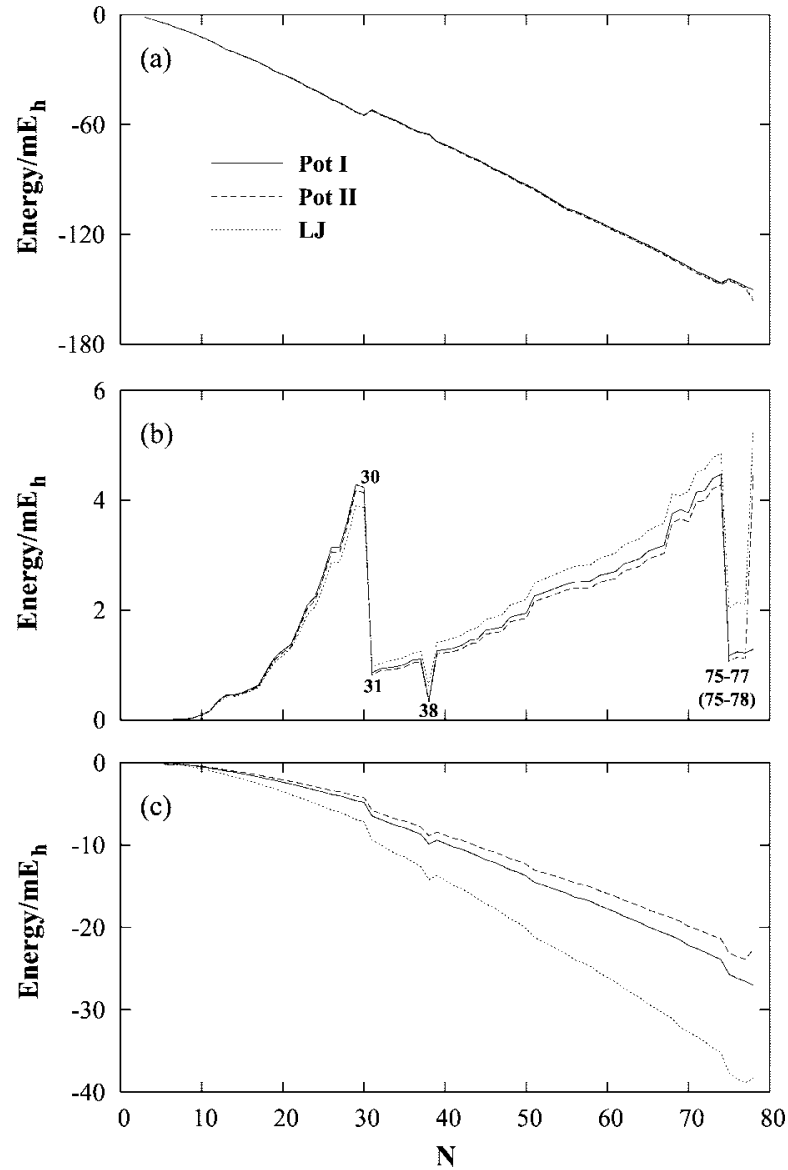

Figure 4. The three contributions to the energy of the cluster according to eq 11 as a function of the size of cluster: (a) nearest-neighbor contribution, $E_{\mathrm{nn}}$; (b) strain energy, $E_{\mathrm{strain}}$; (c) non-nearest-neighbor component, $E_{\mathrm{n}-\mathrm{nn}}$. The lines represent the results for Pot I (solid), Pot II (dashed), and Lennard-Jones (dotted). Major discontinuities are indicated by the corresponding values of $N$ in panel (b); values in parentheses are only for the case of Pot I. See the text.

number of nearest-neighbor distances (which leads to more stable structures); for $N=31$, however, the value of $E_{\text {strain }}$ that would result from an increase of $E_{\mathrm{nn}}$ is too high, that is, leading to an unfavorable geometry. This competition between $E_{\text {strain }}$ and $E_{\mathrm{nn}}$ originates the well-known structural changes for $N=38$ ( $\operatorname{Ar}_{38}$ has octahedral geometry) and $N=$ 75-77 $\left(\mathrm{Ar}_{75}, \mathrm{Ar}_{76}\right.$, and $\mathrm{Ar}_{77}$ have decahedral geometry) for which less-strained structures become more stable than Mackay icosahedron-type ones. Because of this, the number of non-nearest-neighbor distances has an extra increase, and hence, the $E_{\mathrm{n}-\mathrm{nn}}$ curve drops down for those nuclearities (Figure 4c).

Although the $E_{\mathrm{n}-\mathrm{nn}}$ component is expected to have a minor contribution to the structure of the global minimum, ${ }^{48}$ Figure $4 \mathrm{c}$ shows that it becomes more important as the number of atoms increases or the potential presents a more pronounced long-range tail. Specifically, non-nearest-neighbor interactions lead to a contraction of nearest-neighbor distances from the corresponding pair potential equilibrium value (whose effect increases with the range of the potential), ${ }^{50}$ and hence, they have a certain influence in obtaining more compact structures, as discussed above. In addition, non-nearest-neighbor interactions are known to play also a role in lowering the energy of the hexagonal close-packed (hcp) lattices in relation to the face-centered cubic (fcc) ones for LJ potentials. ${ }^{51}$ In fact, the hyperradius of global minimum structures represented 

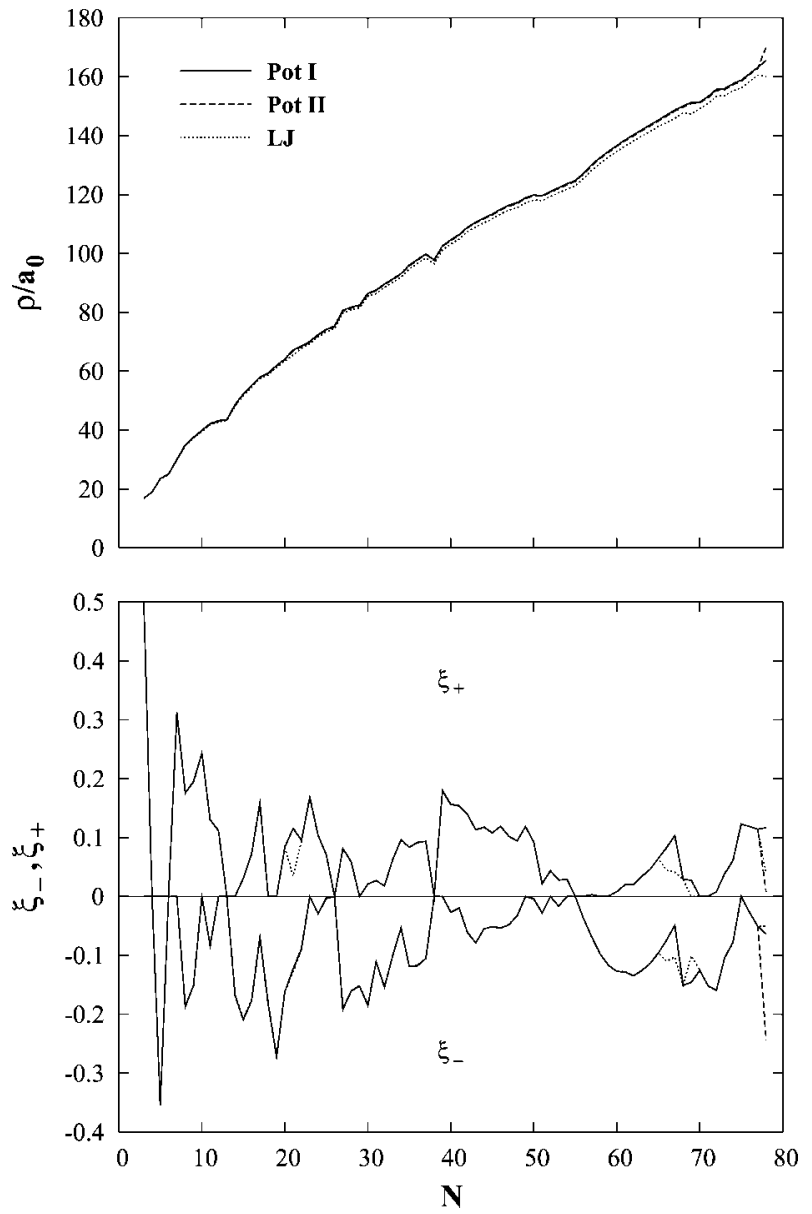

Figure 5. Dependence of hyperradius (upper panel) and deformation indices $\xi_{-}$and $\xi_{+}$(lower panel) on the number of atoms in the cluster. Note that lines below (above) zero in the lower panel are for $\xi_{-}\left(\xi_{+}\right)$ since the deformation indices always fulfill the relations $\xi_{-} \leq 0$ and $\xi_{+} \geq 0$; hence, when $\xi_{-}$and $\xi_{+}$are simultaneously zero, the two corresponding curves touch each other, and the structure is a spherical top. For clarity, the curves for Pot III and Pot IV are not plotted, but they virtually coincide with those for Pot I. See the text.

as a function of $N$ in Figure 5 shows that the LJ potential leads to the most compact structures (lowest values of the hyperradius), which is in agreement with the fact that $\mathrm{LJ}$ is more long-ranged than the other two pair potentials (see Figure 1). Note that the hyperradius $(\rho)$ arises in the hyperspherical coordinates representation and can be related to the moments of inertia of the system $\left(I_{1}, I_{2}\right.$, and $\left.I_{3}\right)$ through the expression ${ }^{52,53}$

$$
\rho^{2}=\frac{I_{1}+I_{2}+I_{3}}{2 M_{N}}
$$

where $M_{N}$ is the total mass of the cluster. Whereas $\rho$ may represent a measure of the compactness of the cluster, its shape is better visualized by the deformation indices ${ }^{52}$

$$
\begin{gathered}
\xi_{+}=\frac{I_{1}-I_{2}}{M_{N} \rho^{2}} \\
\xi_{-}=\frac{I_{3}-I_{2}}{M_{N} \rho^{2}}
\end{gathered}
$$

where one has assumed that $I_{1} \geq I_{2} \geq I_{3}$ and, hence, $\xi_{-} \leq 0$ and $\xi_{+} \geq 0$. On the basis of the values of these deformation indices, one may classify the argon clusters as spherical tops
( $\left.\xi_{-}=\xi_{+}=0\right)$, prolate tops $\left(\xi_{-}<0\right.$ and $\xi_{+}=0$ ), oblate tops $\left(\xi_{+}>0\right.$ and $\left.\xi_{-}=0\right)$, and asymmetric tops $\left(\xi_{-}<0\right.$ and $\xi_{+}>$ 0 ), as described by Aquilanti and collaborators. ${ }^{52}$ In turn, we represent the deformation indices as a function of the number of argon atoms in the lower panel of Figure 5. Note that two sets of lines (for each potential) are shown in this lower panel; one represents $\xi_{+}$and never goes below zero, while the other

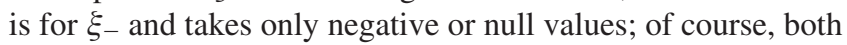
lines touch each other for spherical top structures. For the pair potentials considered in Figure 5, all present spherical tops are at $N=4,6,13,26,38$, and 55; prolate tops arise for $N=$ 5, 14, 18, 19, 29, 56, 58, 69 (only for LJ), 70, 71, and 78 (only for Pot II), while oblate top structures are the $N=3,7,10,12,23,39,49,52,54$, and 75 ones. The remaining clusters of argon considered in this work are asymmetric tops. It is worth noting in Figure 5 the general agreement between Pot I, Pot II, and LJ. In the case of Pot I and Pot II, the only exception arises for $\mathrm{Ar}_{78}$, where the first potential leads to an asymmetric shape global minimum structure (with $C_{1}$ symmetry) while the second one origins a prolate top (with $C_{s}$ symmetry); although having $C_{s}$ symmetry (like for Pot II), the $N=78 \mathrm{LJ}$ cluster is an asymmetric top (like that for Pot I). Besides this, more exceptions arise for the comparison of LJ with Pot I (or Pot II): $N=21,66,67$, and 69. As mentioned above, $\operatorname{Ar}_{69}$ is a prolate (asymmetric) top for the LJ (Pot I and Pot II) potential(s), showing $C_{5 v}\left(C_{s}\right)$ symmetry. Conversely, $\operatorname{Ar}_{21}, \operatorname{Ar}_{66}$, and $\operatorname{Ar}_{67}$ are asymmetric tops for all of the pair potentials, though presenting different magnitudes for the deformation indices of clusters modeled by LJ and both Pot I and Pot II; both $\mathrm{Ar}_{21}$ and $\operatorname{Ar}_{66}$ show $C_{1}$ symmetry for all three potentials, while $\operatorname{Ar}_{67}$ belongs to the $C_{s}\left(C_{2}\right)$ point group in the case of $\mathrm{LJ}$ (Pot I and Pot II).

3.2. Three-Body Contribution. One of the goals of the present work stems from the evaluation of the three-body effects in the structure and energy of the global minima of argon clusters. Although not shown in Figure 3 for clarity, the curves for the average binding energy, sublimation energy, and second energy difference for Pot III and Pot IV essentially resemble the corresponding ones for Pot II. Thus, the main energetic features are not significantly modified by including three-body interactions in the potential of argon clusters up to $\mathrm{Ar}_{78}$. In addition, we can say (although not represented in Figure 5) that the curves of the hyperradius and shape parameters for both Pot III and Pot IV virtually coincide (within the scale of the plots) with those for Pot I (and also for Pot II, excluding $N=$ 78). This result prompts us to conclude that the three-body part of the interaction only slightly perturbs the structural properties (shape and compactness) of the global minima, at least in the range of $N=3-78$. This is an expected outcome since the three-body term of the potential is very small in comparison with the two-body contribution (see above).

Figure 6 displays the contribution of the Axilrod-Teller-Muto triple-dipole interaction for the total energy of argon clusters in the range of $3 \leq N \leq 78$. It is apparent from this figure that the Axilrod-Teller-Muto contribution for the total energy of each global minima is quite small (not reaching $2 \%$ of the total energy). The general trend consists of a fast increase of the threebody contribution up to $N$ around 30; then, it drops down at $N$ $=31$, followed by a slower increase for larger clusters. However, small oscillations occur in the curves of Figure 6 for particular nuclearities, the maxima being associated with less compact icosahedral structures (e.g., $\mathrm{Ar}_{13}$ or $\mathrm{Ar}_{55}$ ), while the minima appear for structures with a certain degree of compactness like the nonicosahedral $\mathrm{Ar}_{38}$ cluster. Note that the triple- 


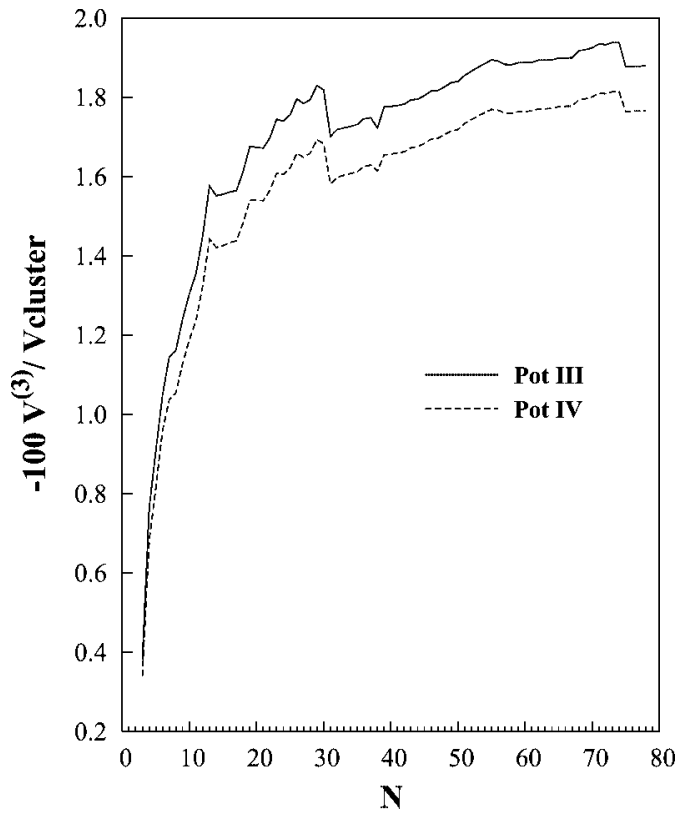

Figure 6. Contribution of the Axilrod-Teller-Muto three-body term to the total energy of argon clusters modeled by Pot III (solid line) and Pot IV (dashed line). Values are given in percentages. See the text.

dipole interaction is generally destabilizing for triangles with only acute angles, which is more likely to occur for compact structures. Another factor that weakens the triple-dipole interactions is the average number of nearest-neighbor atoms, which may partly explain the aforementioned reduction in the threebody contribution at $\mathrm{Ar}_{31}$ and the minima of the curves, for instance, at $\mathrm{Ar}_{21}$ and $\mathrm{Ar}_{24}$; the average number of nearestneighbor atoms is $\sim 3.71$ for $\mathrm{Ar}_{31}$ (in comparison with $\sim 4.03$ for $\mathrm{Ar}_{30}$ and $\sim 3.75$ for $\mathrm{Ar}_{32}$ ), 3.62 for $\mathrm{Ar}_{21}$ (in comparison with $\sim 3.60$ for $\mathrm{Ar}_{20}$ and $\sim 3.68$ for $\mathrm{Ar}_{22}$ ), and $\sim 3.79$ for $\mathrm{Ar}_{24}$ (in comparison with $\sim 3.78$ for $\mathrm{Ar}_{23}$ and $\sim 3.84$ for $\mathrm{Ar}_{25}$ ).

The contribution of three-body interactions at small cluster sizes contrasts with the expected value of $\sim 10 \%$ at the bulk. ${ }^{54}$ Indeed, surface effects, which are responsible for a great reduction of three-body terms, become less important as the number of argon atoms increases. We observe such a trend in Figure 2, although the three-body contribution is far away from the bulk limit. In a similar study but using LJ pair potentials and $C_{\mathrm{DDD}}=516 E_{\mathrm{h}} a_{0}^{9}$ in the Axilrod-Teller-Muto terms (eq $5)$, Wales ${ }^{55}$ has also obtained a nonlinear increase of the change in energy from the many-body to the two-body potential with increasing LJ energy. He also found ${ }^{55}$ that the three-body contribution rises from $\sim 2.6 \%$ for $\mathrm{Ar}_{5}$ to $\sim 5.1 \%$ for $\mathrm{Ar}_{55}$. It is worth noting that the discrepancy between the results of Wales and those from the present work may be due to the different values used in each case for $C_{\mathrm{DDD}}$ as well as the distinct twobody potentials.

Finally, it is interesting to observe in Figure 6 that the inclusion of a damping function to account for charge overlap effects in the triple-dipole term (Pot IV) slightly reduces the three-body contribution for the total energy of the argon clusters. However, such a reduction appears to be similar for all sizes studied; therefore, the curves are essentially parallel to each other.

3.3. Local versus Global Optimization. One important point that arises when studying larger clusters is to know whether a simpler potential (e.g., the LJ potential) may lead to the same global minima structures as those from an accurate function
TABLE 4: Energy Differences between the Putative Minima of Pot I and Those Obtained from the Corresponding LJ and Pot II Structures through Local Optimization

\begin{tabular}{ccc}
\hline cluster & $E_{\text {Pot I }}-E_{\mathrm{LJ}}^{\mathrm{opt}} / m E_{\mathrm{h}}$ & $E_{\mathrm{Pot} \mathrm{I}}-E_{\mathrm{Pot} \mathrm{II}}^{\mathrm{opp}} / m E_{\mathrm{h}}$ \\
\hline $\mathrm{Ar}_{21}$ & $-7.34(-3)$ & \\
$\mathrm{Ar}_{66}$ & $-8.19(-3)$ & \\
$\mathrm{Ar}_{67}$ & $-5.09(-3)$ & \\
$\mathrm{Ar}_{69}$ & $-4.72(-3)$ & $-1.66(-1)$ \\
$\mathrm{Ar}_{78}$ & $-1.08(-1)$ &
\end{tabular}

(e.g., Pot I in the context of this work), which is expected to be rather complicated and, hence, computationally demanding (see Table 1). If so, such a function may be used instead in the global minimization procedure, and the global minimum of the accurate function is then easily obtained by performing a small number of steps of a local search algorithm. Of course, this kind of scheme leads to a strong reduction of the computational effort of the global minimization.

In the context of silicon cluster optimization, Hartke ${ }^{56}$ has proposed a guiding function that is continuously improved by including more accurate information (e.g., ab initio energies) along the minimization procedure. Although more rigorous than the scheme suggested above for two-body potentials, the kind of approach proposed by Hartke ${ }^{56}$ is quite expensive and probably unnecessary for nonbonding rare gas clusters. Thus, we have performed a local optimization for $\operatorname{Ar}_{N}(N=3-78)$ modeled by function Pot I, taking the corresponding LJ cluster geometry ${ }^{46}$ as the starting point; the minima are always reached within a maximum number of 30 iterations. Then, we performed a similar local optimization but giving the global minima geometries obtained with Pot II as starting points. Table 4 shows the differences between the putative global minimum for Pot I and those obtained from the corresponding structures for LJ and Pot II in the unsuccessful cases. It is worth noting that one can obtain the global minima of Pot I through local optimization of the global minimum structures of Pot II, except for $N=78$; note that both the hyperradius and shape indices of $\mathrm{Ar}_{78}$ are different between Pot I and Pot II (see Figure 5), which is an indication that the two global minima cannot interconvert through local optimization. Less efficiency is obtained with LJ that fails the endeavor for $N=21,66,67,69$, and 78. Once again, we observe differences in the shape indices at those sizes between LJ and Pot I (or Pot II); see also section 3.1. However, the major difference in Table 4 occurs for $N=78$. Then, we represent by two perspective views in Figure 7 the corresponding global minimum structures for $\mathrm{Ar}_{78}$. It is clear from this figure that LJ, Pot I, and Pot II potentials lead to rather different structures, and hence, the impossibility of interconversion among them through local optimization is not surprising. We further note that the $N=78$ clusters present different values of nearestneighbor distances for all of the pair potentials (342 for LJ, 332 for Pot I, and 343 for Pot II), which is additional evidence of their structural difference.

A similar local optimization procedure has been carried out for Pot III and Pot IV. Since the three-body contribution included in Pot III and Pot IV is very small (see section 3.2), we wonder whether one can use the global minimum structures of pair potentials as starting points to obtain the corresponding putative minima of those many-body potentials by local optimization. This goal is achieved for both Pot III and Pot IV by taking as starting points the global minima structures of Pot I. It is interesting to note that the $\mathrm{Ar}_{78}$ global minimum structures for Pot III and Pot IV shown in Figure 7 are quite similar to that of Pot I; in addition, as mentioned in section 3.2, the hyperradius 

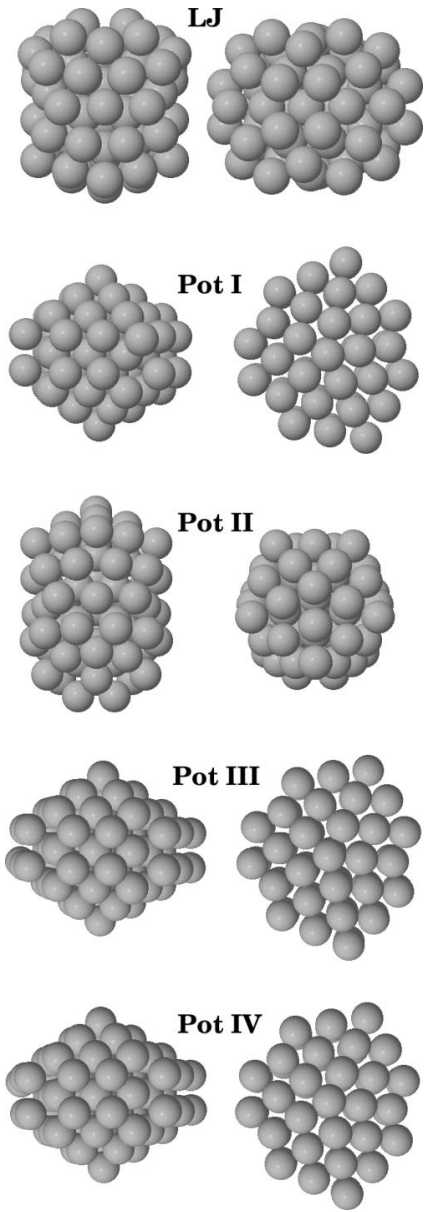

Figure 7. Two perspective views of the $\mathrm{Ar}_{78}$ global minimum structures for potentials LJ, Pot I, Pot II, Pot III, and Pot IV.

and shape parameters for both Pot III and Pot IV essentially coincide with the corresponding ones for Pot I. Conversely, applying the local optimization to the global minimum structures of LJ and Pot II leads to similar results as those met above for the pair potentials (see previous paragraph); LJ fails to give the global minima of Pot III and Pot IV for $N=21,66,67,69$, and 78, while Pot II fails only for $N=78$ (in both cases).

Although the global minimum structure of argon clusters is affected by the particular potential function only for five values of $N$ in the range studied here, one expects that other cases will probably arise for larger nuclearities. We further note that such behavior cannot be extrapolated for other types of clusters. Indeed, it has been shown that the potential has a great influence on the global minima structures of water ${ }^{57-59}$ and lead ${ }^{60}$ clusters, just to mention two important examples.

\section{Conclusions}

We have performed a global minima search of $\operatorname{Ar}_{N}(N=$ 3-78) clusters by using our GA and four potential energy functions. The performance of the simplest version of the GA has shown to be successful (with a good level of efficiency) in finding out the global minima structures for most of the argon cluster structures for the four potentials investigated in this work. However, for some difficult cases (e.g., $\mathrm{Ar}_{75}, \mathrm{Ar}_{76}$, and $\mathrm{Ar}_{77}$ ), the GA failed to discover the global optima; therefore, we modified the algorithm to accomplish a two-phase local optimization procedure. The first minimization is performed in a penalized potential, which is then followed by optimization of the "true" potential. This improvement offers the possibility to enlarge narrow funnels in the potential energy surface, and it enhances the performance of the GA. This is particularly patented for the double-funnel $\mathrm{Ar}_{38}$ cluster, where the improved version of the GA leads to $100 \%$ of success against $\sim 10 \%$ with the original algorithm. Also, the new GA could discover the global minima of all "difficult" cases for global optimization, that is, $\operatorname{Ar}_{65}$ (with Pot I), $\mathrm{Ar}_{75}, \mathrm{Ar}_{76}, \mathrm{Ar}_{77}$, and $\mathrm{Ar}_{78}$ (with Pot I, Pot III, and Pot IV).

The Rydberg-London pair potential constitutes an improvement in relation to the $\mathrm{LJ}$ function since it guarantees the high accuracy of, for example, the Aziz potential at a lower computational cost. Indeed, the global minima given by Pot II are more reliable than the LJ ones to be used as starting geometries to obtain global minima for other accurate potentials (e.g., Pot I) through local optimization. Excluding the case of $\mathrm{Ar}_{78}$ whose global minima properties are quite distinct between Pot I and Pot II, these potentials lead to very similar energetic and structural features for the studied argon clusters. Conversely, the LJ potential presents distinct global minima structural properties for $\mathrm{Ar}_{21}, \mathrm{Ar}_{66}, \mathrm{Ar}_{67}, \mathrm{Ar}_{69}$, and $\mathrm{Ar}_{78}$; in this context, the hyperradius $(\rho)$ and the shape indices $\left(\xi_{-}\right.$and $\left.\xi_{+}\right)$appear to be the most adequate parameters to characterize the structural differences arising in clusters. Moreover, the LJ potential tends to overestimate (in relation to Pot I and Pot II) the average binding energy, the sublimation energy, and the strain energy for $N>30$, which may be due to the fact that $\mathrm{LJ}$ is the most long ranged pair potential.

The contribution of the triple-dipole three-body interaction to the total energy of the global minima has shown to be always less than $2 \%$, and the correction of the Axilrod-Teller-Muto term for small interatomic distances by including a damping function (Pot IV) even reduces the importance of these terms. Curiously, the application of local optimization to the global minima of Pot I allows one to obtain the corresponding minima of many-body potentials (Pot III and Pot IV). Application of local minimization to the LJ structures fails the global minima of Pot III and Pot IV again for $\mathrm{Ar}_{21}, \mathrm{Ar}_{66}, \mathrm{Ar}_{67}, \mathrm{Ar}_{69}$, and $\mathrm{Ar}_{78}$. Excluding $N=78$, all global minima of Pot III and Pot IV can be obtained through local optimization from the corresponding Pot II structures found with our GA. Thus, due to the performance and the low computational cost, we would recommend the use of the global minima of Pot II (rather than LJ ones) as starting points for searching the corresponding rare gas structures obtained with more accurate potentials (e.g., from $\mathrm{ab}$ initio or DFT calculations).

Acknowledgment. This work was supported by Fundação para a Ciência e Tecnologia, Portugal, under Grant POSC/EIA/ $55951 / 2004$. We are grateful to the John von Neumann Institut für Computing, Jülich, for the provision of supercomputer time on the IBM Regatta p690+ (Project EPG01). We also acknowledge Prof. Szalewicz and collaborators (University of Delaware, U.S.A.) for providing us with the FORTRAN routine of their fitted potential [eqs 2 and 3] and Prof. Aquilanti and Dr. Lombardi (University of Perugia, Italy) for a fruitful discussion about ref 52 .

\section{References and Notes}

(1) Sato, T.; Tsuneda, T.; Hirao, K. Mol. Phys. 2005, 103, 1151.

(2) Tao, J.; Perdew, J. P. J. Chem. Phys. 2005, 122, 114102.

(3) Patkowski, K.; Murdachaew, G.; Fou, C.-M.; Szalewicz, K. Mol. Phys. 2005, 103, 2031.

(4) Slavícek, P.; Kalus, R.; Paska, P.; Odvárková, I.; Hobza, P.; Malijevský, A. J. Chem. Phys. 2003, 119, 2102. 10520 .

5) Cybulski, S. M.; Toczylowski, R. R. J. Chem. Phys. 1999, 111, 
(6) Baccarelli, I.; Gianturco, F. A.; González-Lezana, T.; DelgadoBarrio, G.; Miret-Artés, S.; Villarreal, P. J. Chem. Phys. 2005, 122, 144319.

(7) Aziz, R. A. J. Chem. Phys. 1993, 99, 4518.

(8) Naumkin, F. Y.; Wales, D. J. Mol. Phys. 1999, 96, 1295.

(9) Cahill, K.; Persegian, V. A. J. Chem. Phys. 2004, 121, 10839.

(10) Pereira, F. B. Marques, J. M. C. Leitão, T. Tavares, J. Proceedings of the 2006 IEEE Congress on Evolutionary Computation (CEC), Vancouver; Vols. 1-6, 2006; pp 2270-2277.

(11) Locatelli, M.; Schoen, F. Comput. Optim. Appl. 2002, 21, 55.

(12) Locatelli, M.; Schoen, F. Comput. Optim. Appl. 2003, 26, 173.

(13) Korona, T.; Williams, H. L.; Bukowski, R.; Jeziorski, B.; Szalewicz,

K. J. Chem. Phys. 1997, 106, 5109.

(14) Tang, K. T.; Toennies, J. P. J. Chem. Phys. 1984, 80, 3726.

(15) Herring, C. Rev. Mod. Phys. 1962, 34, 631.

(16) Murdachaew, G.; Szalewicz, K.; Jiang, H.; Bacic, Z. J. Chem. Phys. 2004, 121, 11839 .

(17) Hattig, C.; Hess, B. A. J. Phys. Chem. 1996, 100, 6243.

(18) Thakkar, A. J. J. Chem. Phys. 1988, 89, 2092.

(19) Axilrod, B. M.; Teller, E. J. Chem. Phys. 1943, 11, 299.

(20) Muto, Y. Proc. Phys. Math. Soc. Jpn. 1943, 17, 629.

(21) Axilrod, B. M. J. Chem. Phys. 1951, 19, 719.

(22) Standard, J.; Certain, P. C. J. Chem. Phys. 1985, 83, 3002.

(23) Varandas, A. J. C. Mol. Phys. 1983, 49, 817.

(24) Sachse, T. I.; Tang, K. T.; Toennies, J. P. Chem. Phys. Lett. 2000, 317,346

(25) Horn, T. R.; Gerber, R. B.; Valentini, J. J.; Ratner, M. A. J. Chem. Phys. 1991, 94, 6728.

(26) Hartke, B. Proceedings of the Genetic and Evolutionary Computation Conference, GECCO-2001, San Francisco; Spector, L., Goodman, E., Wu, A., Langdon, W. B., Voigt, H.-M., Gen, M., Sen, S., Dorigo, M., Pezeshk., S., Garzon, M., Burke, E., Eds.; Morgan Kaufmann: Burlington, MA, 2001; p 1284.

(27) Roberts, C.; Johnston, R. L.; Wilson, N. T. Theor. Chem. Acc. 2000, 104, 123.

(28) Nocedal, J. Math. Comput. 1980, 35, 773.

(29) Liu, D.; Nocedal, J. Math. Program. B 1989, 45, 503.

(30) Deaven, D. M.; Ho, K. M. Phys. Rev. Lett. 1995, 75, 288.

(31) Pereira, F. B.; Marques, J. M. C.; Leitão, T.; Tavares, J. Advances in Metaheuristics for Hard Optimization, Springer Natural Computing Series; Siarry, P., Michalewicz, Z., Eds.; Springer: Berlin, 2008; pp 223250.

(32) Hartke, B. Struct. Bonding (Berlin) 2004, 110, 33.

(33) Hoare, M. R.; McInnes, J. Faraday Discuss. Chem. Soc. 1976, 61, 12.

(34) Tsai, C. J.; Jordan, K. D. J. Chem. Phys. 1993, 99, 6957.
(35) Stillinger, F. H. Phys. Rev. E 1999, 59, 48.

(36) Wales, D. J. Energy Landscapes: With Applications to Clusters, Biomolecules and Glasses; Cambridge University Press: Cambridge, U.K., 2003.

(37) Doye, J. P. K.; Miller, M. A.; Wales, D. J. J. Chem. Phys. 1999, 111,8417 .

(38) Doye, J. P. K.; Miller, M. A.; Wales, D. J. J. Chem. Phys. 1999, $110,6896$.

(39) Wales, D. J.; Doye, J. P. K. J. Phys. Chem. A 1997, 101, 5111.

(40) Gregurick, S. K.; Alexander, M. H.; Hartke, B. J. Chem. Phys. 1996, 104, 2684.

(41) Wales, D. J.; Scheraga, H. A. Science 1999, 285, 1368.

(42) Hartke, B. J. Comput. Chem. 1999, $20,1752$.

(43) Doye, J. P. K. Phys. Rev. E 2000, 62, 8753.

(44) Doye, J. P. K.; Leary, R. H.; Locatelli, M.; Schoen, F. INFORMS J. Comput. 2004, 16, 371.

(45) Grosso, A.; Locatelli, M.; Schoen, F. Math. Program. 2007, 110, 373.

(46) The Cambridge Cluster Database. http://brian.ch.cam.ac.uk/ccd.html (accessed in December 2007).

(47) Lordeiro, R. A.; Guimarães, F. F. G.; Belchior, J. C.; Johnston,

R. L. Int. J. Quantum Chem. 2003, 95, 112.

(48) Doye, J. P. K.; Wales, D. J.; Berry, R. S. J. Chem. Phys. 1995, $103,4234$.

(49) Doye, J. P. K.; Wales, D. J. J. Chem. Soc., Faraday Trans. 1997, $93,4233$.

(50) Hoare, M. R.; Pal, P. Nat. Phys. Sci. 1972, 236, 35.

(51) Kihara, T.; Koba, S. J. Phys. Soc. Jpn. 1952, 7, 348.

(52) Aquilanti, V.; Lombardi, A.; Yurtsever, E. Phys. Chem. Chem. Phys. 2002, 4, 5040 .

(53) Aquilanti, V.; Lombardi, A.; Sevryuk, M. B. J. Chem. Phys. 2004, 121,5579 .

(54) Johnston, R. L. Atomic and Molecular Clusters; Taylor \& Francis: London, 2002.

(55) Wales, D. J. J. Am. Chem. Soc. 1990, 112, 7908.

(56) Hartke, B. Chem. Phys. Lett. 1996, 258, 144.

(57) Wales, D. J.; Hodges, M. P. Chem. Phys. Lett. 1998, 286, 65.

(58) James, T.; Wales, D. J.; Hernandez-Rojas, J. Chem. Phys. Lett. 2005, 415,302 .

(59) Silva, E. S. A.; Duarte, H. A.; Belchior, J. C. Chem. Phys. 2006, $323,553$.

(60) Doye, J. P. K. Comput. Mater. Sci. 2006, 35, 227.

JP711918T 